\title{
Limited joint mobility
}

National Diabetes Information Clearinghouse (NDIC)

\section{Source}

National Diabetes Information Clearinghouse (U.S.). (2009). The diabetes dictionary. [Bethesda, Md.]: U.S. Dept. of Health and Human Services, National Institutes of Health, National Institute of Diabetes and Digestive and Kidney Diseases, National Diabetes Information Clearinghouse.

A condition in which the joints swell and the skin of the hand becomes thick, tight, and waxy, making the joints less able to move. It may affect the fingers and arms as well as other joints in the body. 